hep-th/0403050

SLAC-PUB-10324

\title{
Non-Singular Solutions for S-branes
}

\author{
Gregory Jones $^{\dagger}$, Alexander Maloney* and Andrew Strominger ${ }^{\dagger}$
}

\begin{abstract}
Exact, non-singular, time-dependent solutions of Maxwell-Einstein gravity with and without dilatons are constructed by double Wick rotating a variety of static, axisymmetric solutions. This procedure transforms arrays of charged or neutral black holes into s-brane (spacelike brane) solutions, i.e. extended, short-lived spacelike defects. Along the way, new static solutions corresponding to arrays of alternating-charge Reissner-Nordstrom black holes, as well as their dilatonic generalizations, are found. Their double Wick rotation yields s-brane solutions which are periodic in imaginary time and potential large- $\mathrm{N}$ duals for the creation/decay of unstable D-branes in string theory.
\end{abstract}

$\dagger$ Department of Physics, Harvard University, Cambridge, MA 02138

* SLAC and Department of Physics, Stanford University, Stanford, CA 94309 


\section{Contents}

1. Introduction . . . . . . . . . . . . . . . . . . . . . . . . . . . . . . . . . 1

2. S-Branes from Black Diholes . . . . . . . . . . . . . . . . . . . . . . 3

2.1. Time Dependent Axisymmetric Einstein-Maxwell Solutions . . . . . . . . . . . 3

2.2. Double Wick Rotation of the Black Dihole . . . . . . . . . . . . . . . . . . . 5

2.3. Adding a Dilaton . . . . . . . . . . . . . . . . . . . . . . . . . . . . . 9

3. Neutral S-Brane Solutions . . . . . . . . . . . . . . . . . . . . . . . . . . . 9

3.1. Double Wick Rotated Black Hole Pair . . . . . . . . . . . . . . . . . . . . 10

3.2. Double Wick-Rotated Black Hole Array . . . . . . . . . . . . . . . . . . . . 11

4. Alternating Charge S-brane Array . . . . . . . . . . . . . . . . . . . . . . . . 12

4.1. Static Alternating-Charge Extremal Black Hole Array . . . . . . . . . . . . . . 12

4.2. Properties . . . . . . . . . . . . . . . . . . . . . . . . . . . . 15

4.3. Double Wick Rotation . . . . . . . . . . . . . . . . . . . . . . . . . . . 16

5. Embedding into String Theory . . . . . . . . . . . . . . . . . . . . . . . . . 16

\section{Introduction}

Nearly a century after the discovery of general relativity, new exact solutions continue to be found. In this paper we construct several families of exact, time-dependent solutions with and without electromagnetic fields and/or dilatons. The solutions are of the s-brane (spacelike brane) type [1], describing a shell of radiation coming in from infinity and creating an unstable brane which subsequently decays. They are of special interest because, in a variety of examples, they are singularity-free (outside horizons) and periodic in imaginary time. In addition, they potentially provide large $N$ duals of unstable D-brane creation/decay in string theory. A variety of other s-brane gravity solutions can be found in [2]6].

The s-brane solutions herein are generated by applying a trick of "double Wick rotation" to static solutions [7]. Typically, a Wick rotation $z \rightarrow i t$ is applied to a Killing direction parameterized by a coordinate $z$ to obtain a static, Lorentzian solution from a Euclidean one. The Killing symmetry implies that the metric is $z$-independent and hence remains real under $z \rightarrow i t$. However, this reality is guaranteed by the weaker condition of a discrete symmetry $z \rightarrow-z$, implying that the metric contains no odd powers of $z$, as well as a suitable condition on the electromagnetic field if present. In this more general context Wick rotation leads to time-dependent solutions. Of course there is no guarantee that the resulting solutions will be singularity-free in general. 
We apply this trick to several interesting static solutions in the literature, including the recently understood black dihole solution [8] representing a pair of oppositely charged black holes and the Israel-Khan infinite line array of neutral black holes [9, 10]. They are Wick rotated twice to obtain a new Lorentzian solution: first along the static time direction

$$
\tau \rightarrow i y
$$

to obtain a Euclidean solution and then again along the spacelike discrete symmetry direction (the axis of the black holes)

$$
z \rightarrow i t
$$

to obtain a new Lorentzian solution. Interestingly enough, while the original black dihole solution has conical "strut" singularities, these are moved off into the complex plane and do not appear in the singularity-free s-brane solution.

Neither of the solutions so obtained quite corresponds to the type of s-brane most naturally arising in string theory 1 These are related by double Wick rotation to a (previously unknown) solution describing a static linear array of alternating-charge black holes. The linearized versions of these solutions were found in [12] using Sen's open string boundary state [13] for the creation/decay of an unstable D-brane. Here we construct an exact non-linear solution describing just such an array, and find that (unlike the dihole case) it is free of strut singularities. The double Wick rotation of this solution is then the nonlinear generalization of the linearized s-brane solutions in [12]. A salient property of these solutions, desired for a connection to string theory and distinguishing them from previous s-brane gravity solutions, is that they are periodic in imaginary time. They bear roughly the same relation to the unstable D-brane creation/decay, that the black $p$-brane solutions [14] bear to ordinary stable D-branes. Hence they may be relevant for a time-dependent large $N$ duality.

This paper is organized as follows. Section 2 begins with a brief review of the Weyl formalism for axisymmetric solutions of the Einstein-Maxwell equations. Emparan's black dihole solution is then described, and Wick-rotated to a smooth s-brane solution. The s-charge is computed and found to be nonzero. Dilatonic generalizations are described. In section 3 we find that double Wick rotation of the Israel-Khan solution for a neutral black hole pair (with strut singularities) leads to a smooth s-brane solution. We also

1 The black dihole s-brane corresponds to generalized sD-brane configurations of the type described in [11]. 
construct the double Wick rotation of the (strut-free) Myers neutral black hole array solution to an s-brane solution. In section 4, following a suggestion of Gibbons [15], we construct a static solution corresponding to an alternating array of KK monopoles and antimonopoles, containing a dilaton from dimensional reduction. The dilaton is removed using the general method of [16], yielding a static line array of alternating-charge ReissnerNordstrøm solutions of the pure Einstein-Maxwell theory. We perform the double Wick rotation (1.1), (1.2) and study various properties of the resulting s-brane solution. Finally, in section 5 we discuss the relevance of these gravity solutions to string theory.

\section{S-Branes from Black Diholes}

We will start by reviewing the general construction of static, axisymmetric solutions of Einstein-Maxwell theory in Section 2.1. We will then proceed to describe the simplest examples of double Wick rotated solutions. These are found by starting with static solutions describing a pair of oppositely charged, extremal black holes. As we will show in Section 2.2, the double Wick rotation procedure (1.1), (1.2) transforms this into an exact, time dependent solution that is everywhere smooth and non-singular.

These solutions for extremally charged black holes have a direct string theory interpretation in terms of D-branes. In particular, dilatonic generalizations of these solutions can be lifted to give s6-brane solutions of IIA supergravity, as is described in Section 2.3.

\subsection{Time Dependent Axisymmetric Einstein-Maxwell Solutions}

A static, axisymmetric solution of Einstein-Maxwell theory has a metric of the form

$$
d s^{2}=-e^{X} d \tau^{2}+e^{-X}\left(\rho^{2} d \varphi^{2}+e^{2 \gamma}\left(d \rho^{2}+d z^{2}\right)\right)
$$

and an electrostatic potential

$$
A_{\mu} d x^{\mu}=A d \tau
$$

Here $X, \gamma$ and $A$ are functions of $\rho$ and $z$ only. We will use units $G=1$. Upon double Wick rotation (1.1), (1.2) this becomes a time dependent solutions of the form

$$
\begin{aligned}
d s^{2} & =e^{X} d y^{2}+e^{-X}\left(\rho^{2} d \varphi^{2}+e^{2 \gamma}\left(d \rho^{2}-d t^{2}\right)\right), \\
A_{\mu} d x^{\mu} & =i A d y .
\end{aligned}
$$


In order for the metric and gauge potential to be real, we see that $A$ must be an antisymmetric function of $z$, whereas $e^{X}$ and $e^{2 \gamma}$ must be symmetric.

The sourceless Maxwell equation is

$$
\ddot{A}+A^{\prime \prime}+\frac{1}{\rho} A^{\prime}=\dot{A} \dot{X}+A^{\prime} X^{\prime},
$$

where prime denotes $\partial / \partial \rho$ and dot denotes $\partial / \partial z$. Einstein's equation implies that

$$
\ddot{X}+X^{\prime \prime}+\frac{1}{\rho} X^{\prime}=2 e^{-X}\left(\dot{A}^{2}+A^{\prime 2}\right)
$$

and

$$
\begin{aligned}
& \frac{4}{\rho} \dot{\gamma}=2 X^{\prime} \dot{X}-8 e^{-X} \dot{A} A^{\prime} \\
& \frac{4}{\rho} \gamma^{\prime}=X^{\prime 2}-\dot{X}^{2}+4 e^{-X}\left(\dot{A}^{2}-A^{\prime 2}\right) .
\end{aligned}
$$

Equation (2.6) determines $\gamma$ in terms of $A$ and $X$. One might worry that these overconstrain $\gamma$, but in fact this system is integrable, as can be seen by testing the equality of mixed partials in (2.6). One important feature of (2.6) is that along the $z$-axis (i.e. for $\rho=0), \dot{\gamma}$ vanishes in the absence of sources. Thus any conical singularities that are present along the $z$-axis will be constant. As we will see below, this fact enables us to get rid of conical singularities altogether in certain double Wick rotated geometries.

For future reference, we will note one important symmetry of (2.5)-(2.6). With $A=0$ (pure Einstein theory), they have the symmetry

$$
X \rightarrow-X+2 \log \rho+\text { constant }, \quad \gamma \rightarrow \gamma-X+\log \rho+\text { constant. }
$$

As we will see in section 4 , this transformation of $X$ corresponds to turning off black holes where they had existed on the z-axis, and turning on black holes where they had not existed. The accompanying transformation of $\gamma$ is required by Einstein's equations.

It remains to solve for $X$ and $A$ using (2.4) and (2.5). As was first noted by Weyl [17] 18], these equations are particularly simply when written in terms of a new three dimensional space with coordinates $(\varphi, \rho, z)$ and flat metric

$$
d \hat{s}^{2}=d \rho^{2}+d z^{2}+\rho^{2} d \varphi^{2}
$$

The equations of motion are

$$
\nabla^{2} X=2 e^{-X}|\nabla A|^{2}, \quad \nabla^{2} A=\nabla A \cdot \nabla X
$$

where $\nabla$ is the covariant derivative with respect to $d \hat{s}^{2}$.

Although beguilingly simple, these equations are difficult to solve analytically when $A \neq 0$. The dihole solution of the next section is one of few such exact solutions that admits a sensible black hole interpretation. 


\subsection{Double Wick Rotation of the Black Dihole}

The black dihole solution

$$
\begin{aligned}
d s^{2} & =-e^{X} d \tau^{2}+e^{-X}\left(\rho^{2} d \varphi^{2}+e^{2 \gamma}\left(d \rho^{2}+d z^{2}\right)\right), \quad A_{\mu} d x^{\mu}=A d \tau \\
e^{X} & =\left[\frac{\left(R_{+}+R_{-}\right)^{2}-4 m^{2}-\frac{k^{2}}{m^{2}+k^{2}}\left(R_{+}-R_{-}\right)^{2}}{\left(R_{+}+R_{-}+2 m\right)^{2}-\frac{k^{2}}{m^{2}+k^{2}}\left(R_{+}-R_{-}\right)^{2}}\right]^{2} \\
e^{2 \gamma} & =\left[\frac{\left(R_{+}+R_{-}\right)^{2}-4 m^{2}-\frac{k^{2}}{m^{2}+k^{2}}\left(R_{+}-R_{-}\right)^{2}}{4 R_{+} R_{-}}\right]^{4} \\
A & =-\frac{4 m k}{\sqrt{m^{2}+k^{2}}} \frac{R_{+}-R_{-}}{\left(R_{+}+R_{-}+2 m\right)^{2}-\frac{k^{2}}{m^{2}+k^{2}}}\left(R_{+}-R_{-}\right)^{2} \\
R_{ \pm} & =\sqrt{\rho^{2}+\left(z \pm \sqrt{m^{2}+k^{2}}\right)^{2}}
\end{aligned}
$$

has been studied by many authors [8, 16, 19]. We will summarize here only a few salient features. The solution (2.10) describes a static configuration of two extremal black holes of equal mass and opposite charge. The solution is written in terms of parameters $m$ and $k ; 2 m$ is the total mass of the system and the black holes are located at $z= \pm \sqrt{m^{2}+k^{2}}$, $\rho=0$. The black holes are extremal in the sense that their horizons are degenerate. The metric has conical singularities extending along the $z$-axis, as may be seen from the limiting form of the metric in the $(\rho, \varphi)$ plane at small $\rho$ and $-\sqrt{m^{2}+k^{2}}<z<\sqrt{m^{2}+k^{2}}$

$$
e^{-X}\left(\left(\frac{k^{2}}{m^{2}+k^{2}}\right)^{4} d \rho^{2}+\rho^{2} d \varphi^{2}\right) .
$$

The standard identification $\varphi \simeq \varphi+2 \pi$ leads to a conical singularity on the interval $-\sqrt{m^{2}+k^{2}}<z<\sqrt{m^{2}+k^{2}}$ extending between the two black holes - this may be thought of as a strut balancing the attractive gravitational and electric forces between the two black holes [8]. This singularity passes through the origin $z=0$, so would lead to a singular solution after the double Wick rotation (11.1), (1.2). We will avoid this problem by noting that the equation of motion (2.6) fixes $\gamma$ only up to a constant of integration. Thus we are free to subtract the constant

$$
\gamma \rightarrow \gamma-2 \log \frac{k^{2}}{m^{2}+k^{2}} .
$$

With this choice, conical deficits are located instead on the semi-infinite intervals $z<$ $-\sqrt{m^{2}+k^{2}}$ and $z>\sqrt{m^{2}+k^{2}}$. This corresponds to hanging the black holes from infinity 
by cosmic strings rather than inserting a strut between them. The subtraction (2.12) is equivalent to adopting the non-standard periodicity for $\varphi$

$$
\varphi \simeq \varphi+2 \pi\left(\frac{k^{2}}{m^{2}+k^{2}}\right)^{2}
$$

Applying the double Wick rotation (1.1), (1.2) gives a time-dependent solution, which we will write as

$$
d s^{2}=e^{-X}\left(\rho^{2} d \varphi^{2}+e^{2 \gamma}\left(d \rho^{2}-d t^{2}\right)\right)+e^{X} d y^{2}, \quad A_{\mu} d x^{\mu}=A d y
$$

where

$$
\begin{aligned}
e^{X} & =\left[\frac{(\operatorname{Re} R)^{2}-m^{2}+\frac{k^{2}}{m^{2}+k^{2}}(\operatorname{Im} R)^{2}}{(\operatorname{Re} R+m)^{2}+\frac{k^{2}}{m^{2}+k^{2}}(\operatorname{Im} R)^{2}}\right]^{2} \\
e^{2 \gamma} & =\left(\frac{m^{2}+k^{2}}{k^{2}}\right)^{4}\left[\frac{(\operatorname{Re} R)^{2}-m^{2}+\frac{k^{2}}{m^{2}+k^{2}}(\operatorname{Im} R)^{2}}{|R|^{2}}\right]^{4} \\
A & =2 m k \sqrt{m^{2}+k^{2}} \frac{\operatorname{Im} R}{\left.\left(k^{2}+m^{2}\right)(\operatorname{Re} R+m)^{2}+k^{2}(\operatorname{Im} R)\right)^{2}} .
\end{aligned}
$$

Here we've defined the complex distance to one of the sourcesl

$$
R=\sqrt{\rho^{2}-\left(t+i \sqrt{m^{2}+k^{2}}\right)^{2}} .
$$

It is straightforward to verify that the metric and gauge potential are smooth and nonsingular for all real values of $t$ and $\rho$.

This solution has translational Killing symmetry $\partial_{y}$ in addition to the usual rotational vector $\partial_{\varphi}$, so resembles the creation and subsequent decay of a 1-brane whose spatial direction extends in the $y$ direction. Suppressing the $y$ direction, the solution describes a spherical wave of gravitational and electromagnetic flux that is localized around the light cone $\rho^{2}=t^{2}$. 3 The solution is asymptotically flat either at large radius or in the far past or future. The gravitational potential $g_{t t}$, the warp factor $e^{X}$ and the potential $A$ are shown in Figure 1 for typical values of $k$ and $m$.

2 This definition $R=\sqrt{\rho^{2}+m^{2}+k^{2}-t^{2}-2 i t \sqrt{m^{2}+k^{2}}}$ involves a branch prescription; we will analytically continue from $t=0$ and note that the radicand traces a parabola which does not intersect the standard branch cut, on the negative real axis.

3 This solution has a translational symmetry in the $y$ direction, so may naturally be thought of as a spherical wave in the other $2+1$ dimensions. It therefore resembles the $2+1$ dimensional supergravity solutions of [20] describing the decay of $\mathbf{C} / \mathbf{Z}_{n}$ singularities. 
a)

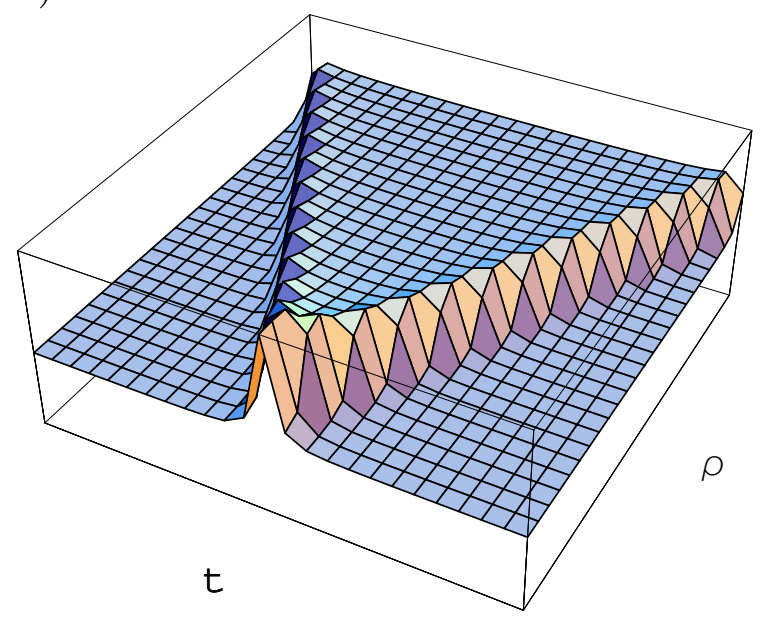

c)

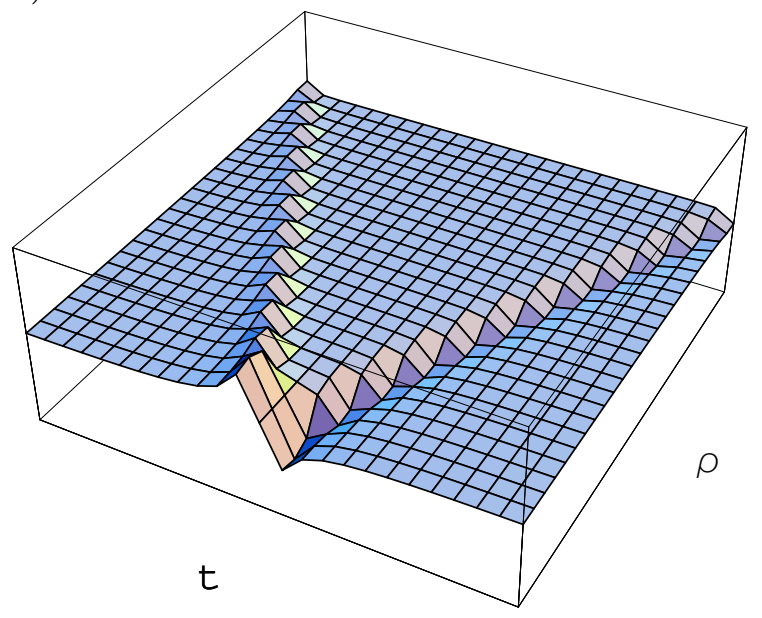

b)

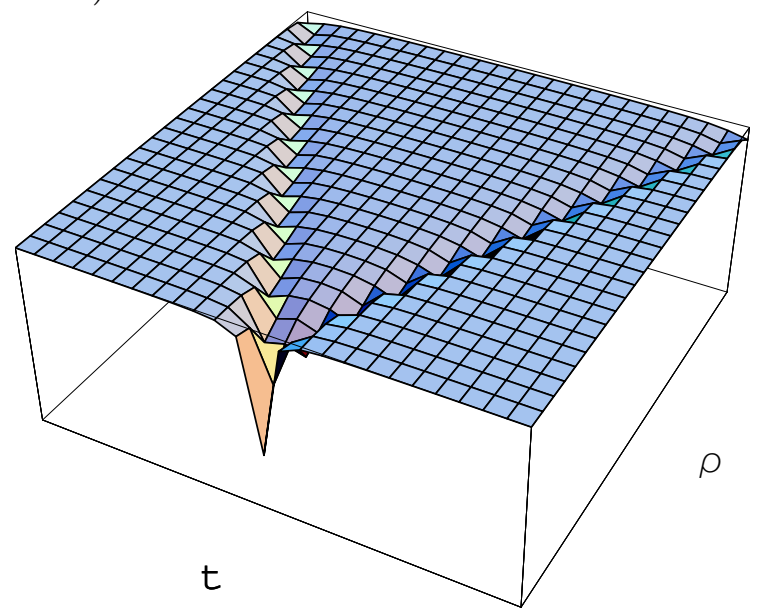

Fig. 1: Various components of the black dihole S-brane solution: (a) the gravitational potential $g_{t t}$, (b) the warp factor $e^{X}$, and (c) the gauge potential $A$. All components are non-singular.

Outside the light cone $\rho^{2}=t^{2}$, the fields asymptote to Minkowski space. As one crosses the light cone the gravitational potential $g_{t t}$ and the gauge field $A$ jump, indicating that an observer feels gravitational and electromagnetic forces, as is typically the case for s-brane solutions. There is a nonzero conserved s-charge found by integrating $\frac{1}{4 \pi} * F$ along a complete transverse spacelike slice (which we can take to be the $\rho \varphi$ plane). It is sufficient to compute $F=\partial A / \partial t d t \wedge d y+\cdots$ and evaluate the charge at time $t=0$. We find

$$
\begin{aligned}
Q_{s} & =\frac{1}{4 \pi} \int \frac{\left(\sqrt{\rho^{2}+m^{2}+k^{2}}-m\right)^{2} 2 m k}{\left(\rho^{2}+k^{2}\right)^{2}\left(\rho^{2}+m^{2}+k^{2}\right)^{1 / 2}} \rho d \rho \wedge d \varphi \\
& =\frac{m}{k}\left(\sqrt{m^{2}+k^{2}}+m\right) .
\end{aligned}
$$


This is the same as the charge magnitude $Q$ of either extremal black hole in the original black dihole geometry (2.10). To see that this must be the case, consider computing $Q$ by integrating $\frac{1}{4 \pi} * F$ on a smooth topological sphere $S$ surrounding the black hole at $z=\sqrt{m^{2}+k^{2}}$. Now deform and enlarge $S$ so that it is the boundary of a half-ball; $S$ comprises a disk in the $z=0$ plane and a half-sphere centered at $z=0, \rho=0$. Taking the limit where the radius of the disk and half-sphere goes to infinity, the disk becomes the $(\rho, \varphi)$ plane and the flux through the half-sphere goes to zero. The quantity $\left.F_{t z}\right|_{z=0} d t \wedge d z$ is unaffected (up to a conventional sign) by the double Wick rotation, and the result (2.17) follows.

This geometry has an interesting limit where the parameter $m$ is large compared to $k, \rho$, and $t$. Specifically, we take $m \rightarrow m / \lambda,(k, \rho, t) \rightarrow(k, \rho, t) / \lambda^{2}$, and $y \rightarrow \lambda^{2} y$ where $\lambda \rightarrow \infty$. The physical significance of this limit will be discussed shortly. Expanding the functions in (2.14)-(2.15), we find in this limit

$$
\begin{aligned}
d s^{2} & =\left(\frac{k^{2}+\rho^{2}}{4 m^{2}}\right)^{2}\left[d y^{2}+\left(\frac{4 m^{2}}{k^{2}}\right)^{4}\left(-d t^{2}+d \rho^{2}\right)\right]+\left(\frac{4 m^{2}}{k^{2}+\rho^{2}}\right)^{2} \rho^{2} d \varphi^{2} \\
A & =-\left(k t / 2 m^{2}\right) d y, \quad F=-\left(k / 2 m^{2}\right) d t \wedge d y
\end{aligned}
$$

This is the Melvin solution describing a constant electric field pointing in the $y$ direction, with physical strength $\vec{E} \cdot \hat{y}=\frac{k}{2 m^{2}}\left(\frac{k^{2}}{k^{2}+\rho^{2}}\right)^{2}$. These solutions have been well studied in the context of string theory [21] - they are typically written in terms of the dual field strength

$$
* F=-\frac{8 k m^{2} \rho d \rho \wedge d \varphi}{\left(k^{2}+\rho^{2}\right)^{2}},
$$

which represents a constant magnetic field.

The appearance of the Melvin solution has a natural physical interpretation. Consider the geometry before the double Wick rotation. The black holes are located at $z= \pm \sqrt{m^{2}+k^{2}}$, which becomes $z= \pm \lambda m$ in our limit. As $\lambda \rightarrow \infty$ the black holes are pulled off to infinity and we are left with the electric flux tube - which is described by the Melvin universe - running between them. This relation between static black diholes and the Melvin universe was studied in [22]. The same idea applies to the double Wick rotated geometry, since the Melvin solution (2.18) is preserved under double Wick rotation up to a rescaling of $t$. Here, as the sources recede away along the imaginary $t$ axis the solution becomes static.

From this discussion, we see that for $k \ll m$, the solution (2.15) can be thought of as the creation and subsequent decay of an electric Melvin flux tube located near $\rho=0$. 


\subsection{Adding a Dilaton}

The dihole solutions described above have straightforward dilatonic generalizations, which were studied by [16,22, 23, 24]. The details of the double Wick rotation are essentially the same as in the non-dilatonic case, so we will just state the results.

The s-brane solution of Einstein-Maxwell-Dilaton theory with dilaton coupling $\alpha$ and extremal electric sources is

$$
\begin{aligned}
d s^{2} & =e^{-X /\left(1+\alpha^{2}\right)}\left(\rho^{2} d \varphi^{2}+e^{2 \gamma /\left(1+\alpha^{2}\right)}\left(d \rho^{2}-d t^{2}\right)\right)+e^{X /\left(1+\alpha^{2}\right)} d y^{2} \\
A_{\mu} d x^{\mu} & =\frac{1}{\sqrt{1+\alpha^{2}}} A d y, \quad e^{2 \phi}=e^{\alpha X /\left(1+\alpha^{2}\right)}
\end{aligned}
$$

where $X, \gamma$ and $A$ are as in (2.15). This solution is smooth and non-singular for all real $\rho$ and $t$. The structure of this solution is very similar to the configuration described above. These dilatonic solutions can be lifted to give solutions of IIA string theory. In this case the closed string configurations are sourced by Euclidean D6-branes, and are related to the creation and subsequent decay of an unstable D7-brane.

As one might expect, the $m \rightarrow \infty$ limit of this solution gives the electric Melvin solution of string theory. This is the electric dual of the fluxbrane solutions written down by [21].

\section{Neutral S-Brane Solutions}

In this section we study the general time-dependent solutions found by Wick rotating an axisymmetric collection of sources on the $z$-axis with no charges. Unlike the solutions

of the previous section, these configurations are not sourced by extremal objects on the $z$-axis, and hence do not have a simple string theory interpretation in terms of Euclidean D-branes. Nevertheless, they are interesting new, non-singular, time-dependent solutions of general relativity and string theory.

We will first describe the Wick rotation of a pair of neutral black holes - the details of this construction are similar to that of the dihole in the previous section. Next, we apply this procedure to the periodic array of neutral black holes described by Myers [10]. This yields an s-brane type solution that is explicitly periodic in imaginary time. 


\subsection{Double Wick Rotated Black Hole Pair}

When there are no electromagnetic fields $(A=0)$, the equations of motion $(2.9)$ simplify considerably. Axisymmetric solutions to the sourceless Einstein equations are found by solving Laplace's equation in three dimensions,

$$
\nabla^{2} X=0
$$

The general solution is found by specifying a density of sources $b(z)$ distributed along the $z$-axis. In this case we can immediately write down the solution

$$
X(\rho, z)=-2 \int_{-\infty}^{\infty} d z^{\prime} \frac{b\left(z^{\prime}\right)}{\sqrt{\rho^{2}+\left(z-z^{\prime}\right)^{2}}}
$$

and proceed to solve for $\gamma$ using (2.6). To avoid naked singularities, the linear density $b(z)$ must typically equal $1 / 2$ on some line segments and be zero elsewhere [18]. A simple solution is given by placing a rod source of length $2 m$ and density $b=1 / 2$ on the $z$-axis. This gives

$$
\begin{aligned}
e^{X} & =\frac{R_{+}+r_{+}-2 m}{R_{+}+r_{+}+2 m}, & e^{2 \gamma} & =\frac{\left(R_{+}+r_{+}\right)^{2}-4 m^{2}}{4 R_{+} r_{+}} \\
R_{+} & =\sqrt{\rho^{2}+(z+m)^{2}}, & r_{+} & =\sqrt{\rho^{2}+(z-m)^{2}}
\end{aligned}
$$

In fact, this is precisely the Schwarzschild solution with mass $m$. The coordinate transformation between (3.3) and the usual Schwarzschild coordinates may be found in [16].

Laplace's equation is linear, so we can easily construct from this multi-black hole solutions by superposing copies of the Schwarzschild solution (3.3). These are known as the Israel-Khan solutions [9]. For example, the solution for two separated black holes is

$$
\begin{aligned}
e^{X} & =\frac{\left(R_{+}+r_{+}-2 m\right)\left(R_{-}+r_{-}-2 m\right)}{\left(R_{+}+r_{+}+2 m\right)\left(R_{-}+r_{-}+2 m\right)} \\
R_{ \pm} & =\sqrt{\rho^{2}+(z \pm(k+m))^{2}}, \quad r_{ \pm}=\sqrt{\rho^{2}+(z \pm(k-m))^{2}}
\end{aligned}
$$

Here the two black holes sources are located on the intervals $-k-m<z<-k+m$ and $k-m<z<k+m$. In this case the double Wick-rotated solution is

$$
\begin{aligned}
d s^{2} & =e^{-X}\left(\rho^{2} d \varphi^{2}+e^{2 \gamma}\left(d \rho^{2}-d t^{2}\right)\right)+e^{X} d y^{2} \\
e^{X} & =\left|\frac{R+r-2 m}{R+r+2 m}\right|^{2} \\
R & =\sqrt{\rho^{2}-(t+i(k+m))^{2}}, \quad r=\sqrt{\rho^{2}-(t+i(k-m))^{2}} .
\end{aligned}
$$


The expression for $e^{2 \gamma}$ can be found explicitly [10]:

$$
e^{2 \gamma}=\left(\frac{k^{2}}{k^{2}-m^{2}}\right)^{2} \frac{\left(|R|^{2}+\rho^{2}-t^{2}-(k+m)^{2}\right)\left(|r|^{2}+\rho^{2}-t^{2}-(k-m)^{2}\right)}{\left|R \bar{r}-t^{2}-2 m i t-k^{2}+m^{2}+\rho^{2}\right|^{2}}
$$

As in the dihole case, we have chosen the prefactor so that the Wick rotated solution is smooth and non-singular for all real $t, y$ and $\rho$. With this choice of $\gamma$, the original solution (3.4) has conical singularities located on the semi-infinite intervals $z<-m-k, z>m+k$

- these are interpreted as cosmic strings that balance the attractive force between the black holes.

\subsection{Double Wick-Rotated Black Hole Array}

We can apply this procedure to find the solution for an infinite array of Schwarzschild black holes, with rod-centers at $z=(2 p+1) k$ for integers $p$, and mass $m$ :

$$
\begin{aligned}
e^{X}= & \prod_{p=-\infty}^{\infty} \frac{R_{p}+r_{p}-2 m}{R_{p}+r_{p}+2 m} \\
e^{2 \gamma}= & \prod_{p, q=-\infty}^{\infty} \frac{r_{q} R_{p}+(z-(2 q+1) k-m)(z-(2 p+1) k+m)+\rho^{2}}{r_{q} r_{p}+(z-(2 q+1) k-m)(z-(2 p+1) k-m)+\rho^{2}} \times \\
& \frac{R_{q} r_{p}+(z-(2 q+1) k+m)(z-(2 p+1) k-m)+\rho^{2}}{R_{q} R_{p}+(z-(2 q+1) k+m)(z-(2 p+1) k+m)+\rho^{2}} \\
R_{p}= & \sqrt{\rho^{2}+(z-(2 p+1) k+m)^{2}}, \quad r_{p}=\sqrt{\rho^{2}+(z-(2 p+1) k-m)^{2}} .
\end{aligned}
$$

These sums formally diverge, but may be regularized using a procedure employed by Myers [10]. For solutions of pure gravity, the equations of motion (2.6) and (3.1) determine $\gamma$ and $X$ only up to constants of integration, so we may subtract off the divergent constants

$$
\begin{aligned}
X_{\text {divergent }} & =\sum_{p=-\infty}^{\infty} \ln \left(\frac{1-m /|2 p+1| k}{1+m /|2 p+1| k}\right) \\
\gamma_{\text {divergent }} & =\sum_{p \geq 0, q \leq-1} \ln \left(1-m^{2} /(p-q)^{2} k^{2}\right) .
\end{aligned}
$$

Any further finite subtraction in $X$ is up to us; this is the usual ambiguity in the potential from a linearly extended source. However, the subtraction in $\gamma$ has been chosen such that the solution has no conical singularities and is smooth and non-singular. The ability to 
eliminate all conical singularities in the geometry is not surprising, since for this configuration the forces between the black holes are balanced. The double Wick rotated solution is then

$$
\begin{aligned}
& e^{X}=e^{-X_{\text {divergent }}} \prod_{p=-\infty}^{\infty} \frac{R_{p}+r_{p}-2 m}{R_{p}+r_{p}+2 m} \\
& e^{2 \gamma}=e^{-2 \gamma_{\text {divergent }}} \prod_{p, q=-\infty}^{\infty} \frac{r_{q} R_{p}-(t+i(2 q+1) k+i m)(t+i(2 p+1) k-i m)+\rho^{2}}{r_{q} r_{p}-(t+i(2 q+1) k+i m)(t+i(2 p+1) k+i m)+\rho^{2}} \times \\
& \frac{R_{q} r_{p}-(t+i(2 q+1) k-i m)(t+i(2 p+1) k+i m)+\rho^{2}}{R_{q} R_{p}-(t+i(2 q+1) k-i m)(t+i(2 p+1) k-i m)+\rho^{2}} \\
& R_{p}=\sqrt{\rho^{2}-(t+i(2 p+1) k+i m)^{2}}, \quad r_{p}=\sqrt{\rho^{2}-(t+i(2 p+1) k-i m)^{2}} .
\end{aligned}
$$

\section{Alternating Charge S-brane Array}

In this section we find a new solution of Einstein-Maxwell theory in $3+1$ dimensions (with or without a dilaton) which corresponds to a non-supersymmetric, infinite array of alternating-charge extremal black holes. We then double Wick rotate this solution, yielding an exact solution for s-branes of the type encountered in string theory [12]. In particular, these solutions are periodic in imaginary time. We thank Gary Gibbons for suggestions leading to the construction of this section.

\subsection{Static Alternating-Charge Extremal Black Hole Array}

We begin with the Israel-Khan solution with sources of length $k$ centered at $z=$ $(2 p+1) k$ given in (3.9). Analytically continuing the time coordinate $\tau \rightarrow i x^{5}$ gives the Euclidean solution

$$
d s^{2}=e^{X}\left(d x^{5}\right)^{2}+e^{-X}\left(e^{2 \gamma}\left(d \rho^{2}+d z^{2}\right)+\rho^{2} d \varphi^{2}\right)
$$

In analogy with Euclidean Schwarzschild, we anticipate that identifying $x^{5}$ on a thermal circle $x^{5} \simeq x^{5}+2 \pi R$ for some $R$ will yield a non-singular geometry. The black hole horizons are replaced by 2 -surfaces where $\partial / \partial x^{5}$ vanishes; these surfaces are termed 'bolts' [25], and are along the rods at $\rho=0$ where $e^{X}$ vanishes. We have

$$
R=\lim _{\rho \rightarrow 0} \rho e^{\gamma} / e^{X} .
$$


We see that $R$ depends on the infinite constant we subtracted from $X$; we can subtract from $X$ and scale $R$ such that $R e^{X}$ remains constant, and maintain nonsingular bolts.

However we wish to do something a bit different-namely a twisted KK compactification, as in [26]. We first add a flat time direction (as in the construction of the KK monopole 27,28]) to get

$$
d s^{2}=-d \tau^{2}+e^{-X}\left(e^{2 \gamma}\left(d \rho^{2}+d z^{2}\right)+\rho^{2} d \varphi^{2}\right)+e^{X}\left(d x^{5}\right)^{2} .
$$

Consider the Killing vector $K=R \partial_{5}+\partial_{\varphi}$; its fixed points are the north and south poles of each horizon. Such isolated fixed points are known as nuts or antinuts, depending on the relative orientation of the $S O(2) \times S O(2)$ rotation induced by the Killing vector in the tangent space at the nut/antinut [25]. The north pole will be a (self-dual) nut, and the south pole an (anti-self-dual) antinut [25]; after Kaluza-Klein reduction, these will become a magnetic monopole and antimonopole, respectively.

An equivalent procedure is to change coordinates to $\tilde{\varphi}=\varphi-x^{5} / R$ and then reduce along orbits of $\partial_{5}$ with $\tilde{\varphi}$ held fixed. This turns out to be notationally simpler. In the new coordinate, the metric is

$$
d s^{2}=-d \tau^{2}+e^{-X}\left(e^{2 \gamma}\left(d \rho^{2}+d z^{2}\right)+\rho^{2} d \tilde{\varphi}^{2}\right)+2 e^{-X} \frac{\rho^{2}}{R} d \tilde{\varphi} d x^{5}+\left(e^{X}+\frac{e^{-X} \rho^{2}}{R^{2}}\right)\left(d x^{5}\right)^{2} .
$$

Now, perform KK compactification with $\partial / \partial x^{5}$ as the Killing vector. Using

$$
d \hat{s}^{2}=e^{-4 \phi / \sqrt{3}}\left(d x^{5}+2 A_{\mu} d x^{\mu}\right)^{2}+e^{2 \phi / \sqrt{3}} g_{\mu \nu} d x^{\mu} d x^{\nu}
$$

which yields the Einstein frame action

$$
S=\frac{1}{16 \pi G_{4}} \int d^{4} x \sqrt{-g}\left(R-2(\nabla \phi)^{2}-e^{-2 \sqrt{3} \phi} F^{2}\right),
$$

we get

$$
\begin{aligned}
d s^{2} & =\left(e^{X}+e^{-X} \frac{\rho^{2}}{R^{2}}\right)^{1 / 2}\left(-d \tau^{2}+e^{-X} e^{2 \gamma}\left(d \rho^{2}+d z^{2}\right)+\frac{\rho^{2}}{e^{X}+e^{-X} \rho^{2} / R^{2}} d \tilde{\varphi}^{2}\right) \\
A_{\mu} d x^{\mu} & =\frac{\rho^{2} R}{2\left(\rho^{2}+R^{2} e^{2 X}\right)} d \tilde{\varphi} \\
e^{-4 \phi / \sqrt{3}} & =e^{X}+e^{-X} \frac{\rho^{2}}{R^{2}} .
\end{aligned}
$$

4 This observation is related to the fact [8] that the black dihole solution with appropriate dilaton coupling is related to a Schwarzchild instanton. 
We wish to look for possible singularities on the symmetry axis $\rho=0$. In the sections of the $z$-axis where there had been no rods, $e^{X} \rightarrow$ (nonzero) as $\rho \rightarrow 0$, and the relevant part of the metric is

$$
e^{-X} e^{2 \gamma} d \rho^{2}+\frac{\rho^{2}}{e^{X}+e^{-X} \rho^{2} / R^{2}} d \tilde{\varphi}^{2} \rightarrow e^{-X}\left(e^{2 \gamma} d \rho^{2}+\rho^{2} d \tilde{\varphi}^{2}\right)
$$

so the absence of singularities in the Israel-Khan solution guarantees their absence here. On the other hand, in the sections where there had been rods, $e^{X} \sim \rho^{2}$ and the metric looks like

$$
e^{-X} e^{2 \gamma} d \rho^{2}+\frac{\rho^{2}}{e^{X}+e^{-X} \rho^{2} / R^{2}} d \tilde{\varphi}^{2} \rightarrow e^{-X} e^{2 \gamma} d \rho^{2}+e^{X} R^{2} d \tilde{\varphi}^{2}
$$

Now we see that the same choice of $x^{5}$ periodicity (4.2) which makes the Euclideanized Israel-Khan solution regular at the horizons, ensures that our twisted KK-reduced solution is regular along the rod locations.

Although the $5 \mathrm{D}$ geometry is smooth, there are singularities in the $4 \mathrm{D}$ description at the rod endpoints. These are locally as considered in [27,28 and correspond to KK monopoles or anti-monopoles. For $z$ at endpoints of a rod, $e^{X} \sim \rho$ as $\rho \rightarrow 0$, whereas for $z$ in the interior of a rod, $e^{X} \sim \rho^{2}$ as $\rho \rightarrow 0$. From this we compute that $g_{t t} \rightarrow 0$ as $\rho^{1 / 2}$ at the endpoints while $g_{t t} \rightarrow$ (nonzero) on the interior of the rods. This signifies the presence of extremal $\alpha=\sqrt{3}$ dilatonic black holes at the endpoints of the rods. There are Dirac strings running along the rods, joining each monopole-antimonopole pair. The monopole charge can be computed from the Dirac string: we simply place a sphere around the monopole with a neighborhood of the Dirac string deleted. This yields

$$
Q=\frac{1}{4 \pi} \int_{\text {deleted sphere }} d A=\frac{1}{4 \pi} \oint_{\text {small circle }} A \rightarrow \frac{1}{4 \pi} \int_{0}^{2 \pi} \frac{R}{2} d \tilde{\varphi}=R / 4
$$

where we have computed the line integral in the $\rho \rightarrow 0$ limit.

Using the general method of [16], one can remove the dilaton from (4.7) to get a solution of the pure Einstein-Maxwell theory. In this manner we find

$$
\begin{aligned}
d s_{\mathrm{EM}}^{2} & =\left(e^{X}+e^{-X} \rho^{2} / R^{2}\right)^{2}\left(-d \tau^{2}+e^{-4 X} e^{8 \gamma}\left(d \rho^{2}+d z^{2}\right)\right)+\left(e^{X}+e^{-X} \rho^{2} / R^{2}\right)^{-2} \rho^{2} d \tilde{\varphi}^{2} \\
A_{\mathrm{EM}} & =2 A
\end{aligned}
$$

This solution describes a static linear array of extremal Reissner-Nordstrøm black holes with alternating charges $\pm R / 2$. Again the solution is free of singularities because the opposing forces on each black hole cancel without the need for struts. 


\subsection{Properties}

The geometry (4.7) has the symmetry

$$
X \rightarrow-X+2 \log (\rho / R), \quad \gamma \rightarrow \gamma-X+\log (\rho / R)
$$

times charge conjugation. The transformation of $X$ and $\gamma$ can be interpreted using (2.7), (3.2) as turning off the rods that are present, and turning on rods where there were none. This however is equivalent to to shifting $z \rightarrow z+2 k$. Thus (4.7) has the symmetry $z \rightarrow z+2 k$ times charge conjugation. The regions on the $z$-axis where there used to be rods are identical to the regions on the $z$-axis where there had been no rods.

Next we write down the $\rho \rightarrow \infty$ asymptotic geometry and use it to compute the magnetic flux through the $(\rho, \tilde{\varphi})$-plane. Since $X \sim \log (\rho / R)$, from (2.6) we get $\gamma \sim$ $\frac{1}{4} \log \rho+C$, for $C$ a constant. We find

$$
\begin{aligned}
d s^{2} & \sim\left(\frac{2 \rho}{R}\right)^{1 / 2}\left(-d t^{2}+\left(\frac{R}{\rho}\right)^{1 / 2} e^{2 C}\left(d \rho^{2}+d z^{2}\right)+\frac{\rho R}{2} d \tilde{\varphi}^{2}\right) \\
A & \sim \frac{R}{4} d \tilde{\varphi} \\
e^{-4 \phi / \sqrt{3}} & \sim \frac{2 \rho}{R} .
\end{aligned}
$$

The gauge field in the $\rho \rightarrow \infty$ limit is a Wilson line. From Stokes' theorem we can compute the flux through the $(\rho, \tilde{\varphi})$-plane at $z=0$, to be $R \pi / 2$. After dividing by $4 \pi$, this becomes the s-charge $Q_{s}=R / 8$ of the double Wick rotated solution. In the computation of the flux through the $(\rho, \tilde{\varphi})$-plane for $z=k$, one encounters the Dirac string at $\rho=0$ yielding a flux of $-R \pi / 2$.

Naively the solution is described by two parameters, $k$ and $R$. Actually, the two solutions $(k, R)$ and $(\kappa k, R)$ are identical; sending $\rho \rightarrow \kappa \rho$ and $z \rightarrow \kappa z$ in the second solution and using $\exp (X(\kappa \rho, \kappa z ; \kappa k))=\kappa \exp (X(\rho, z ; k))$, we see that they differ only by a coordinate transformation. So we only have one parameter, $R$, which is proportional to the charge. 


\subsection{Double Wick Rotation}

The double Wick rotation (11.1), (11.2) turns the solutions (4.7) and (4.10) into s-branes in the usual way. For example, the dilatonic array (4.7) becomes

$$
\begin{aligned}
d s^{2} & =\left(e^{X}+e^{-X} \rho^{2} / R^{2}\right)^{1 / 2}\left(d y^{2}+e^{-X} e^{2 \gamma}\left(d \rho^{2}-d t^{2}\right)+\frac{\rho^{2}}{e^{X}+e^{-X} \rho^{2} / R^{2}} d \tilde{\varphi}^{2}\right) \\
A_{\mu} d x^{\mu} & =\frac{\rho^{2} R}{2 \rho^{2}+2 R^{2} e^{2 X}} d \tilde{\varphi} \\
e^{-4 \phi / \sqrt{3}} & =e^{X}+e^{-X} \rho^{2} / R^{2} .
\end{aligned}
$$

As above, this is a non-singular, time-dependent solution describing the creation and subsequent decay of an unstable 1-brane in four dimensions.

\section{Embedding into String Theory}

In this section we comment on the potential relevance of these solutions for string theory.

An unstable D-brane in string theory has an open string tachyon on its world volume [29]. A process in which this tachyon is condensed in the far future, but nears the top of its potential for a finite amount of time, describes the creation/decay of an unstable brane. The result is a spacelike region of finite time duration in which open strings can exist, or an s-brane [1]. These objects are of interest because they are spacelike, and therefore time-dependent, versions of the usual string theory D-branes and hence may provide a useful tool for investigations of time-dependent processes in string theory.

The starting point for a concrete description of s-branes is the observation [13] that the open string tachyon profile

$$
T\left(X^{0}\right)=\lambda \cosh \left(X^{0} / \sqrt{\alpha^{\prime}}\right),
$$

where $X^{0}$ is the timelike worldsheet boson, leads to a time-dependent boundary CFT describing the creation and subsequent decay of an unstable brane. The corresponding boundary state sources a closed string configuration, which describes the linearized far field behavior of closed string fields in the presence of a rolling tachyon. For the critical

value of $\lambda=\frac{1}{2}$ this boundary state is related by Wick rotation to an array of sD-branes (i.e. D-branes with a transverse time direction) located at imaginary times $t=i(2 n-1) \sqrt{\alpha^{\prime}}$, 
where $n$ is an integer [11, 12, 13, 30]). The reason for this is simple: the tachyon profile (5.1) may be Wick rotated $X^{0} \rightarrow i X_{E}^{0}$ to give the boundary deformation $\lambda \cos \left(X_{E}^{0} / \sqrt{\alpha^{\prime}}\right)$ describing a periodic array of sD-branes at the critical coupling. Interestingly, despite the fact that there are no D-branes for real values of time when $\lambda=\frac{1}{2}$, the CFT boundary state still describes a highly nontrivial closed string configuration. In superstring theories, these boundary states describe an alternating array of D-branes located at $t=i(4 n-1) \sqrt{\alpha^{\prime}} / \sqrt{2}$ and anti-D-branes at $t=i(4 n+1) \sqrt{\alpha^{\prime}} / \sqrt{2}$. This boundary state contains direct information only about the linearized closed string fields, corresponding to the linearization of the full non-linear solutions described above.

A significant generalization of this construction was found in [11]. Forgetting its origin as a critical rolling tachyon on an unstable brane, the sD-brane array can simply be viewed as a concise rule for constructing a classical closed string field configuration. This rule can be consistently generalized by moving the positions of the sD-branes subject to certain restrictions [11]. In particular one can change the spacing or number of branes.

The relation of the s-brane solutions discussed herein to string theory constructions should now be clear. Consider an unstable D7-brane in IIA string theory, with an open string tachyon of the form (5.1) at the critical value, which is an alternating sD6 - sD̄ 6 array. Now, using the analysis of [11], deform the solution by increasing the imaginary-time spacing between the sD-branes to $k \gg \sqrt{\alpha^{\prime}}$. Next increase the number of initial unstable D-branes to $N$, yielding an array of large $N$ clusters of sD6 and sD̄ 6 branes. In the limit of large $N$ with $g_{s} N$ fixed, this should have a dual gravity description as the product of the $4 \mathrm{D}$ s-brane solution $(4.7)$ with $\mathbf{R}^{6}$.

Note that in motivating this correspondence we have only invoked the standard and very general large $N$ open-closed string duality. In particular we have not introduced any analog of the near-horizon scaling limit [31], which transforms general open-closed string duality into a powerful and practical tool. It would certainly be of great interest to find an analog of this near-horizon scaling in the present time-dependent context.

Among the most interesting sD-brane arrays are those that arise directly from tachyon profiles like (5.1), without deforming the spacing. In this case the sD-branes are separated by imaginary distances of order the string scale - in the closed string description, this

5 With a flat metric in the M-theory frame. Note that D6-branes lift to KK monopoles in M-theory. 
means that massive string states are becoming important [30] 6 . Hence the gravity solutions described here are not trustworthy for such configurations. Nevertheless a study of the limit of small spacing could be interesting.

Finally we note that the constraints of [11] allow a pair of oppositely charged sDbranes at equal imaginary distances from the real time axis. Hence, following the preceding discussion, one can find an embedding of the black dihole s-brane solution (2.15) into string theory.

\section{Acknowledgements}

This work was supported in part by DOE grants DE-FG02-91ER40654 and DE-AC0376SF0015. We are grateful to G. Gibbons, G. Horowitz, F. Leblond, E. Silverstein, and X. Yin for useful conversations.

6 To be more specific, the CFT description of rolling tachyons describes Euclidean branes and anti-branes separated by the imaginary distance $\sqrt{2 \alpha^{\prime}}$. At this distance, the open string tachyon stretching between a brane/anti-brane pair becomes massless, leading to the usual infrared divergence in the partition function. In the closed string language this is interpreted as an ultraviolet divergence coming from very massive string states. 


\section{References}

[1] M. Gutperle and A. Strominger, "Spacelike branes," JHEP 0204, 018 (2002) arXiv:hep-th/0202210.

[2] F. Leblond and A. W. Peet, "SD-brane gravity fields and rolling tachyons," JHEP 0304, 048 (2003) arXiv:hep-th/0303035.

[3] M. Kruczenski, R. C. Myers and A. W. Peet, JHEP 0205, 039 (2002) arXiv:hepth/0204144.

[4] C. M. Chen, D. V. Gal'tsov and M. Gutperle, Phys. Rev. D 66, 024043 (2002) arXiv:hep-th/0204071.

[5] A. Buchel, P. Langfelder and J. Walcher, Annals Phys. 302, 78 (2002) arXiv:hep th/0207235.

[6] C. P. Burgess, F. Quevedo, S. J. Rey, G. Tasinato and I. Zavala, JHEP 0210, 028 (2002) arXiv:hep-th/0207104.

[7] W. B. Bonnor, "Exact Solutions of the Einstein-Maxwell Equations," Zeitschrift für Physik 161, p. 439 (1961).

[8] R. Emparan, "Black diholes," Phys. Rev. D 61, 104009 (2000) arXiv:hep-th/9906160.

[9] W. Israel and K. A. Khan, Nuovo Cim., 33, 331 (1964).

[10] R. C. Myers, "Higher-dimensional black holes in compactified space-times," Phys. Rev. D 35 (2), January 1987, p. 455.

[11] D. Gaiotto, N. Itzhaki and L. Rastelli, "Closed strings as imaginary D-branes," arXiv:hep-th/0304192.

[12] A. Maloney, A. Strominger and X. Yin, "S-brane thermodynamics," JHEP 0310, 048 (2003) arXiv:hep-th/0302146.

[13] A. Sen, "Rolling tachyon," JHEP 0204, 048 (2002) arXiv:hep-th/0203211.

[14] G. T. Horowitz and A. Strominger, Nucl. Phys. B 360, 197 (1991).

[15] G. Gibbons, private communication.

[16] R. Emparan and E. Teo, "Macroscopic and microscopic description of black diholes," Nucl. Phys. B 610, 190 (2001) arXiv:hep-th/0104206.

[17] H. Weyl, Ann. Phys. (Leipzig) 54 (1917) 117

[18] R. Emparan and H. S. Reall, Phys. Rev. D 65, 084025 (2002) arXiv:hep-th/0110258.

[19] E. Teo, "Black diholes in five dimensions," Phys. Rev. D 68, 084003 (2003) arXiv:hepth/0307188].

[20] M. Headrick, "Decay on C/Z(n): Exact supergravity solutions," arXiv:hep-th/0312213.

[21] M. Gutperle and A. Strominger, "Fluxbranes in string theory," JHEP 0106, 035 (2001) arXiv:hep-th/0104136.

[22] R. Emparan and M. Gutperle, "From p-branes to fluxbranes and back," JHEP 0112, 023 (2001) arXiv:hep-th/0111177. 
[23] A. Davidson and E. Gedalin, "Finite Magnetic Flux Tube As A Black And White Dihole," Phys. Lett. B 339, 304 (1994) arXiv:gr-qc/9408006,

[24] Y. C. Liang and E. Teo, "Black diholes with unbalanced magnetic charges," Phys. Rev. D 64 (2001) arXiv:hep-th/0101221.

[25] G. W. Gibbons and S. W. Hawking, "Classification of Gravitational Instanton Symmetries," Commun. Math. Phys. 66 (1979) p. 291.

[26] F. Dowker, J. P. Gauntlett, G. W. Gibbons and G. T. Horowitz, "The Decay of magnetic fields in Kaluza-Klein theory," Phys. Rev. D 52, 6929 (1995) arXiv:hepth/9507143].

[27] D. J. Gross and M. J. Perry, "Magnetic Monopoles in Kaluza-Klein Theories," Nucl. Phys. B226 (1983), p. 29.

[28] R. D. Sorkin, "Kaluza-Klein Monopole," Phys. Rev. Let. 51 2, p. 87.

[29] A. Sen, "Non-BPS states and branes in string theory," arXiv:hep-th/9904207.

[30] N. Lambert, H. Liu and J. Maldacena, "Closed strings from decaying D-branes," arXiv:hep-th/0303139.

[31] J. M. Maldacena, Adv. Theor. Math. Phys. 2, 231 (1998) [Int. J. Theor. Phys. 38, 1113 (1999)] [arXiv:hep-th/9711200]. 